\title{
The mineralogy, geochemistry and health risk assessment of deposited particulate matter (PM) in Kraków, Poland
}

\author{
Agnieszka Gruszecka-Kosowska ${ }^{1}$, Magdalena Wdowin ${ }^{2}$ \\ ${ }^{1}$ AGH University of Science and Technology, Faculty of Geology, Geophysics, and Environmental Protection; \\ al. Mickiewicza 30; 30-059 Krakow, Poland; e-mail:agnieszka.gruszecka@agh.edu.pl \\ ${ }^{2}$ Polish Academy of Sciences, Mineral and Energy Economy Research Institute; \\ ul. Wybickiego 7; 31-261 Krakow, Poland; e-mail:wdowin@min-pan.krakow.pl
}

(C) 2016 Authors. This is an open access publication, which can be used, distributed and reproduced in any medium according to the Creative Commons CC-BY 4.0 License requiring that the original work has been properly cited.

Received: 2016-11-18; accepted: 2017-02-21

\begin{abstract}
One of the most important air contaminants in Krakow is particulate matter (PM), especially during winter months. Deposited PM was sampled between November 2014 and January 2015 and November 2015 and January 2016. The PM deposition amounted to $0.0646 \mathrm{~g} / \mathrm{m}^{2}$ per day in the centre of Krakow and $0.0328 \mathrm{~g} / \mathrm{m}^{2}$ in the northern district of the city. The allowed value of annual dust deposits $\left(0.547 \mathrm{~g} / \mathrm{m}^{2}\right.$ per day) was not exceeded. The XRD and SEM-EDS analysis showed that quartz, calcite, potassium feldspar, plagioclase, kaolinite, and gypsum were the main mineralogical components of the deposited PM. Only in a few samples were illite, dolomite, and apatite observed. Significant concentrations were measured (ICP-MS) for $\mathrm{Ca}, \mathrm{Fe}, \mathrm{Mg}, \mathrm{Al}, \mathrm{K}, \mathrm{Si}, \mathrm{Na}$, and $\mathrm{Mn}$, and high concentrations for $\mathrm{Ti}, \mathrm{Cu}, \mathrm{Zn}, \mathrm{As}, \mathrm{Pb}, \mathrm{Cr}, \mathrm{Ti}, \mathrm{V}, \mathrm{Li}, \mathrm{Sr}$ and $\mathrm{Ni}$. The highest estimated daily intakes (EDI) for resuspended PM, were stated for ingestion exposure pathway than for dermal contact, and finally for inhalation, both for children and adults, as well as, for carcinogenic and non-carcinogenic elements. The mean hazard quotient (HQ) values for all three exposure pathways decreased in the following order: $\mathrm{Fe}>\mathrm{Al}>\mathrm{Zn}>\mathrm{Mn}>\mathrm{Sr}>\mathrm{Cu}$ $>\mathrm{Ba}>\mathrm{Cr}>\mathrm{Pb}>\mathrm{V}>\mathrm{Ni}>\mathrm{Li}>\mathrm{As}>\mathrm{Sn}>\mathrm{Zr}>\mathrm{Co}>\mathrm{Cd}>\mathrm{Be}$, for children and adults. The hazard index values for all elements in each exposure pathway $\left(\mathrm{HI}_{\text {elem }}\right)$ and for single elements in all exposure pathways $\left(\mathrm{HI}_{\text {path }}\right)$ were $<1$, both for children and adults. The risk values from all three exposure pathways were unacceptable in the case of $\mathrm{Cr}$, both for children and adults, and in the case of As for children. The total non-carcinogenic risk $\left(\mathrm{HI}_{\text {total }}\right)$ values in all three exposure pathways for all elements were $<1$, however in case of children the risk value pointed to a low risk level. The total carcinogenic risk $\left(\mathrm{R}_{\text {total }}\right)$ values in all three exposure pathways for As and Cr exceeded the acceptable level, both for children and adults.
\end{abstract}

Keywords: particulate matter, air quality, human health, mineralogical composition, chemical composition

\section{INTRODUCTION}

Particulate matter (PM) is identified as one of the most hazardous air pollutants nowadays in terms of its impact on human health (WHO 2013, EEA 2014). Epidemiological studies have shown that air contamination with PM is responsible for the significant increase in the incidence of allergies (Baldacci et al. 2015), respiratory (Brunekreef \& Holgate 2002, Götschi 2008, Guarnieri \& Balmes 2014, Sarrigannis et al. 2015) and cardiovascular
(Peters et al. 2001, Pope et al. 2002, Brook et al. 2004) system diseases, and increased mortality in populations (Samet et al. 2000, Polichetti et al. 2009, Lipmann et al. 2013, Wang et al. 2014, Lee et al. 2015, Li et al. 2015). Crucial factors are the physical and chemical properties of PM i.e. particle size, size distribution, density, shape, hydrophilic or hydrophobic character (Patra et al. 2016). Dust health effects are caused not only by the penetration of respiratory tract by PM, but they also depend on PM concentration, as well 
as their chemical and mineralogical composition that may augment the related health problems. The size of the PM affects their penetration and deposition (Megido et al. 2016), while chemical composition affects the clearance (Nag et al. 2005, Aleksandropoulou \& Lazaridis 2013) of the human respiratory system. Also important are physiological factors as breathing pattern (Patra et al. 2016). The chemical composition of PM varies widely depending on emission sources, emitted compounds, and chemical reactions taking place in the atmosphere (Rashki et al. 2013, Samara et al. 2016). The presence of exceeded PM levels in the ambient air also has a harmful impact on the environment causing reduced biodiversity, disorders of biological integrity from the level of individuals to ecosystems (Grantz et al. 2003), and may affect climate changes (IPCC 2007, Fuzzi et al. 2015).

Air contamination with PM is a serious environmental and health problem for many countries i.e. China (Shao et al. 2006, Jiming et al. 2007, Kan et al. 2012, Zhou et al. 2015), India (Beig et al. 2013, Pant et al. 2016), Saudi Arabia (Munir et al. 2016), as well as Europe (WHO 2016). According to the most recent WHO report, Krakow occupied no. 11 position on the list of the 50 most polluted cities in EU (WHO 2016).

The pollution with PM under the framework of the State Environmental Monitoring System by the Regional Environmental Protection Inspectorate (WIOŚ) is currently measured in Krakow at eight air-quality monitoring stations (Fig. 1). Fraction PM10 is measured at all monitoring stations, while PM2.5 fraction is measured only at following three stations: Krasińskiego Av., Kurdwanów Housing Estate and Nowa Huta district. Regarding inorganic compounds, chemical analysis of PM include concentrations of arsenic (As), lead $(\mathrm{Pb})$, and cadmium $(\mathrm{Cd})$, in the PM10 fraction, measured at three air-quality monitoring stations (Kurdwanów Housing Estate, Wadów Housing Estate, and Nowa Huta district), as well as, nickel (Ni) in the PM10 fraction that is measured at two air-quality monitoring stations (Kurdwanów Housing Estate and Nowa Huta district) at the moment (WIOŚ 2017).

In the current study the mineralogical and chemical properties of the deposited particulate matter in Krakow were presented for PM samples collected during winter months (2014-2016), when air contamination with PM in Krakow is highest (WIOŚ 2017). Based on this information the health risk arising from resuspension of the PM for children and adults in Krakow was estimated.

\section{MATERIALS AND METHODS}

\section{Sample collection}

The deposited PM samples were collected simultaneously at ten locations between November 2014 and January 2015 and between November 2015 and January 2016. Eight sampling points were located in the city of Krakow (Fig. 1), and two outside of the city: in Mogilany (point 9) and Włosań (point 10 on the map). Dust containers, with the surface area of $0.3 \mathrm{~m}^{2}$ each, were placed at the height of ca. $2 \mathrm{~m}$ above the ground to avoid secondary emission from the ground. Collected deposited PM samples were tested for their mineralogical and chemical composition.

\section{Mineralogical analysis}

Analysis was conducted in the Faculty Laboratory in the Faculty of Geology, Geophysics and Environmental Protection of the AGH University of Science and Technology in Krakow, Poland. Qualitative mineralogical analysis of the mineral composition of the dust samples was conducted by the X-ray Diffraction (XRD) and the Debye-Sherrer powder methods. XRD patterns of all the samples were recorded with a Rigaku MiniFlex 600 $\mathrm{X}$-ray diffractometer, using the following parameters: $\mathrm{CuK} \alpha$ radiation, reflexive graphite monochromator, $40 \mathrm{kV}$ lamp voltage, lamp of $20 \mathrm{~mA}$, $0.05^{\circ} 2 \Theta$ step, and $1 \mathrm{~s} /$ step pulse counting rate. The interplanar distance values obtained from the $\mathrm{X}$-ray diffractograms were used for the purpose of mineral identification of the tested samples, based on the data contained in the ICDD (International Centre for Diffraction Data) catalogue and the XRAYAN software.

Morphological forms and the chemical composition of the deposited PM were obtained, using a Scanning Electron Microscope (SEM) with energy dispersive X-ray spectrometry SEM-EDS FEI Quanta 250 FEG. 




Fig. 1. Deposited particulate matter (PM) at sample-collection locations: 1-10 - deposited PM sample-collection points. Sam-

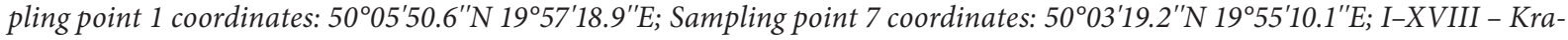
kow district numbers; $A-H$ - air-quality monitoring stations of the Regional Environmental Protection Inspectorate in Krakow (WIOŚ) (A - Złoty Róg Str., B - Piastów Housing Estate, C - Nowa Huta district, D - Krasińskiego Av., E - Dietla Str., F - Kurdwanów Housing Estate, G - Wadów Housing Estate, H - Telimeny Str.)

\section{Chemical analysis}

Analysis was conducted in the Environmental Analysis Laboratory in the Department of Environmental Protection, Faculty of Geology, Geophysics and Environmental Protection of the AGH University of Science and Technology in Krakow, Poland. Samples of deposited PM (points 1 and 7) were mineralized (DigiPREP - SCP Science Utilities, version 3.0) with aqua regia for $2 \mathrm{~h}$ in the temperature $130^{\circ} \mathrm{C}$, according to the US EPA 3050B extraction method (US EPA 1996a). Concentrations of the elements in extracts were determined by Inductively Coupled Plasma Mass Spectroscopy (ICP-MS), using a Perkin Elmer ELAN
6100 apparatus, according to the US EPA method 6020B (US EPA 1998).

\section{Health risk assessment}

Potential exposure pathways arising from resuspended PM episodes are: inhalation, digestion and dermal contact. For all of the analysed elements, the non-carcenogenic risk was calculated, together with carcinogenic risk for As and Cr. The estimated daily intakes (EDI) in three exposure pathways: $\mathrm{PM}$ inhalation $\left(\mathrm{EDI}_{\mathrm{inh}}\right), \mathrm{PM}$ ingestion $\left(\mathrm{EDI}_{\text {ing }}\right)$ and dermal contact with PM (EDI $\left.{ }_{\text {der }}\right)$ were calculated using formulas (1)-(3), respectively (US EPA 1996b, 2001, ATSDR 2005). 


$$
\begin{aligned}
& \mathrm{EDI}_{\text {inh }}=\mathrm{C} \times(\mathrm{InhR} \times \mathrm{EF} \times \mathrm{ED}) / \\
& (\mathrm{PEF} \times \mathrm{BW} \times \mathrm{AT}) \\
& \mathrm{EDI}_{\text {ing }}=\mathrm{C} \times(\mathrm{IngR} \times \mathrm{EF} \times \mathrm{ED} \times \mathrm{CF}) / \\
& (\mathrm{BW} \times \mathrm{AT}) \\
& \mathrm{EDI}_{\text {der }}=\mathrm{C} \times(\mathrm{SA} \times \mathrm{SL} \times \mathrm{ABS} \times \mathrm{EF} \times \mathrm{ED} \times \mathrm{CF}) / \\
& (\mathrm{BW} \times \mathrm{AT})
\end{aligned}
$$

where: $\mathrm{C}$ - exposure point concentration (concentration of the element in deposited PM, this study) $(\mathrm{mg} / \mathrm{kg})$; InhR - inhalation rate, in this study $7.6 \mathrm{~m}^{3} /$ day for children and $20 \mathrm{~m}^{3} /$ day for adults (Liu et al. 2014 vide Van den Berg 1995); IngR ingestion rate, in this study $200 \mathrm{mg} /$ day for children and $100 \mathrm{mg}$ /day for adults (US EPA 2001); EF - exposure frequency, in this study 180 days/year (Fereira-Baptista \& De Miguel 2005); ED - exposure duration; in this study 6 years for children and 70 years for adults; PEF - particle emission factor, in this study $1.36 \mathrm{E}+09 \mathrm{~m}^{3} / \mathrm{kg}$ (US EPA 2001); CF - conversion factor, in this study $10^{-6} \mathrm{~kg} / \mathrm{mg}$ (US EPA 2001); BW - body weight, $15 \mathrm{~kg}$ for children and $70 \mathrm{~kg}$ for adults (Trojanowska \& Świetlik 2016); AT - averaging time, $\mathrm{ED} \times 365$ days; $\mathrm{SA}$ - exposed skin area, in this study $2800 \mathrm{~cm}^{2}$ for children and $5700 \mathrm{~cm}^{2}$ for adults (US EPA 2001); SL - skin adherence factor, in this study $0.2 \mathrm{mg} / \mathrm{cm}^{2}$ for children and $0.07 \mathrm{mg} / \mathrm{cm}^{2}$ for adults (US EPA 2001); ABS dermal absorption factor (unitless) (US EPA 2001).

The carcinogenic risk (R) was calculated using the formula (4) (US EPA 1989):

$\mathrm{R}=\mathrm{EDI} \times \mathrm{SF}$

where: EDI - Estimated Daily Intake (mg/kg body weight per day); SF - slope factor ((mg/kg body weight per day) ${ }^{-1}$ ).

The non-carcinogenic risk was determined using hazard quotient (HQ), calculated using the formula (5) (US EPA 1989):

$\mathrm{HQ}=\mathrm{EDI} / \mathrm{RfD}$

where: EDI - Estimated Daily Intake (mg/kg body weight per day); RfD - Reference Dose (mg/kg body weight per day).

To estimate the total non-carcinogenic risk of many pollutants at the same time, the hazard index (HI) parameter was estimated, in accordance to equitation (6) (US EPA 1989):
$\mathrm{HI}=\mathrm{HQ} 1+\mathrm{HQ} 2+\ldots+\mathrm{HQ} n$

where $1-n$ are specified elements in the deposited PM.

There is no significant risk, if the HI value is $<1$ for non-carcinogenic, and if the $\mathrm{R}$ value is $<1.00 \mathrm{E}-05$ for carcinogenic exposure factors (US EPA 2001, Rozporzadzenie... 2016).

\section{RESULTS AND DISCUSSION}

\section{Particulate matter deposition}

Based on the performed experiments, it was calculated that PM deposition amounted to $0.0646 \mathrm{~g} / \mathrm{m}^{2}$ per day at point 7 (the centre of Krakow) and $0.0328 \mathrm{~g} / \mathrm{m}^{2}$ per day at point 1 (the northern district). The allowed value of annual dust deposit $\left(200 \mathrm{~g} / \mathrm{m}^{2}\right.$ per year and $0.547 \mathrm{~g} / \mathrm{m}^{2}$ per day in Poland; Tomaszewska \& Olszowski 2012) was not exceeded in the winter months of 2015-2016. However, it should be mentioned that the winters when the deposited PM samples were collected were quite mild, thus during more severe winters this deposition should be higher. This could be caused mainly by the higher quantities of burned coal and other fuels, including illegal municipal waste, for heating purposes.

\section{Mineralogical analysis of deposited PM}

Based on XRD analysis, the following mineral phases were identified in all dust samples: quartz, calcite, potassium feldspar, plagioclase, kaolinite, and gypsum (Fig. 2). The presence of illite was noticed in the samples collected at points 5,8 , and 10 ; dolomite at points 1,4 , and 10 ; apatite at points 2 , 5, and 9; and portlandite and gismondine at point 2. Also, hematite was observed in the sample collected at point 5 .

SEM observations indicated that the analysed PM samples contained grains of different sizes representing various morphologies, from spherical to spherical-porous and sharp-edged (Fig. 3). The SEM-EDS analyses identified the presence of mainly amorphous and spherical aluminosilicate glass (fly ash), silica (quartz), iron oxide, mica, feldspars, and chlorides. Besides, fragments of organic matter were observed (pieces of plants, fungi, and algae) (Fig. 3). 


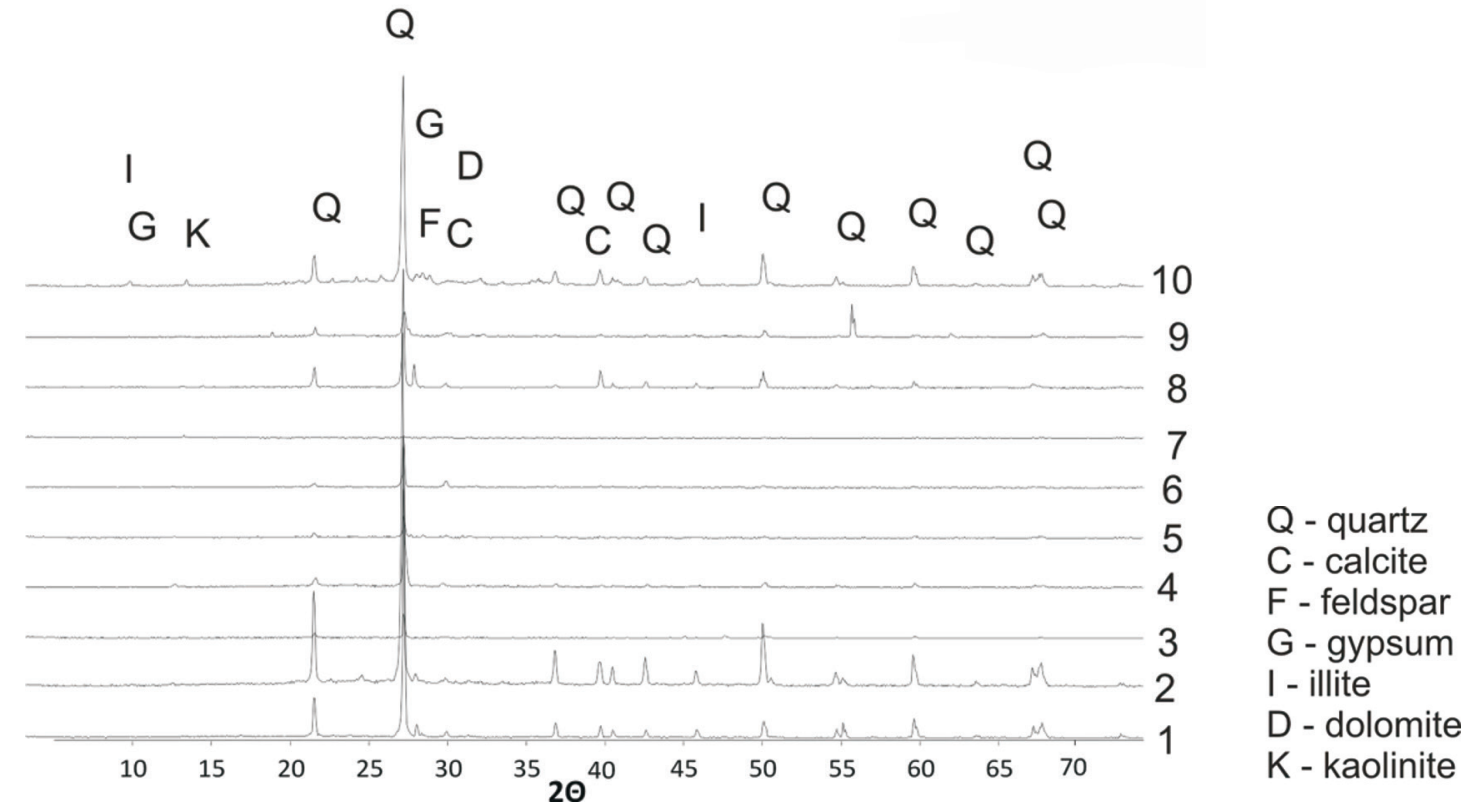

Fig. 2. XRD patterns of the deposited particulate matter (PM): 1-10 - deposited PM sample-collection points

\section{Chemical analysis of deposited PM}

The concentration of selected elements in the deposited particulate matter, investigated using ICP-MS, showed very high concentrations of $\mathrm{Ca}$, $\mathrm{Fe}, \mathrm{Mg}, \mathrm{Al}, \mathrm{K}, \mathrm{Si}, \mathrm{Na}$, and $\mathrm{Mn}$ (Tab. 1). Chemical results are in agreement with mineralogical analysis indicating that the mineralogical composition of dust was dominated by feldspars and aluminosilicates of $\mathrm{K}, \mathrm{Na}, \mathrm{Ca}, \mathrm{Mg}, \mathrm{Fe}$, and $\mathrm{Mn}$. High $\mathrm{Fe}$ concentration resulted from the presence of iron oxides and micas. The high concentrations of $\mathrm{Ti}, \mathrm{Cu}$, and $\mathrm{Zn}$, as well as $\mathrm{As}$, could indicate the dust having originated from the burning of coal (Hulett et al. 1980, Hansen et al. 1981), while high concentrations of $\mathrm{Pb}, \mathrm{Cr}, \mathrm{Ti}$, and $\mathrm{V}$ could indicate the source as being traffic. Also, Ti, Li, and $\mathrm{Sr}$ were observed in large quantities (Tab. 1). A similar mineralogical composition results were obtained by Wilczyńska-Michalik et al. (2015) and Wilczyńska-Michalik \& Michalik (2015). The presence of the above mentioned elements indicates that the emission sources of the deposited PM in Krakow could be: diesel engine emissions (presence of $\mathrm{Al}, \mathrm{Ca}, \mathrm{Cu}, \mathrm{Fe}, \mathrm{Mg}, \mathrm{Mn}, \mathrm{V}, \mathrm{Zn}$ in the $\mathrm{PM})$, gasoline emissions $(\mathrm{Sr}, \mathrm{Cu}, \mathrm{Mn})$, brakes $(\mathrm{Fe}, \mathrm{Cu}, \mathrm{Sn}, \mathrm{Zn})$, tyres (Cd, Co, Cr, $\mathrm{Cu}, \mathrm{Fe}, \mathrm{Mn}$, $\mathrm{Pb})$, road dust ( $\mathrm{Zn}, \mathrm{Al}, \mathrm{K}, \mathrm{Fe}, \mathrm{Na}, \mathrm{Mn})$, industrial sources including metalworking $(\mathrm{Fe}, \mathrm{K}, \mathrm{Na}, \mathrm{Pb}$, Zn) (Sanderson et al. 2014 and references herein).
The concentration of $\mathrm{Ti}$ in the air as claimed by Wilczyńska-Michalik et al. (2014), was not noted in the past and current Ti sources are new building materials and paints. Mineralogical and chemical investigations proved that the composition of atmospheric dust was quite diverse. This would suggest that the PM originated from various sources of emissions (Wilczyńska-Michalik \& Michalik 2015). A similar chemical composition of street dust in Polish cities was stated in the research of Krajewska \& Niesiobędzka 2009, Kiebała et al. 2015, and Trojanowska \& Świetlik 2016.

\section{Health risk assessment}

It can be stated that the risk deriving from air pollution with PM can pose risks for human health. The exposure pathways, chemical and mineralogical composition of PM mentioned above, together with its amount in the air (Patra et al. 2016) and deposition on the ground (Fereira-Baptista \& De Miguel 2005, Liu et al. 2014, Mohmand et al. 2015), and suspension time in the air (Raes et al. 2000, Williams et al. 2002) undoubtedly affect the risk value. The studies of Fuzzi et al. 2015 and Patra et al. 2016 have shown that the inhalation of elevated concentrations of crystalline silica, mica, beryllium, copper, iron, as well as, heavy metals, affects the development of respiratory and cardiovascular diseases, including cancer and premature death. 

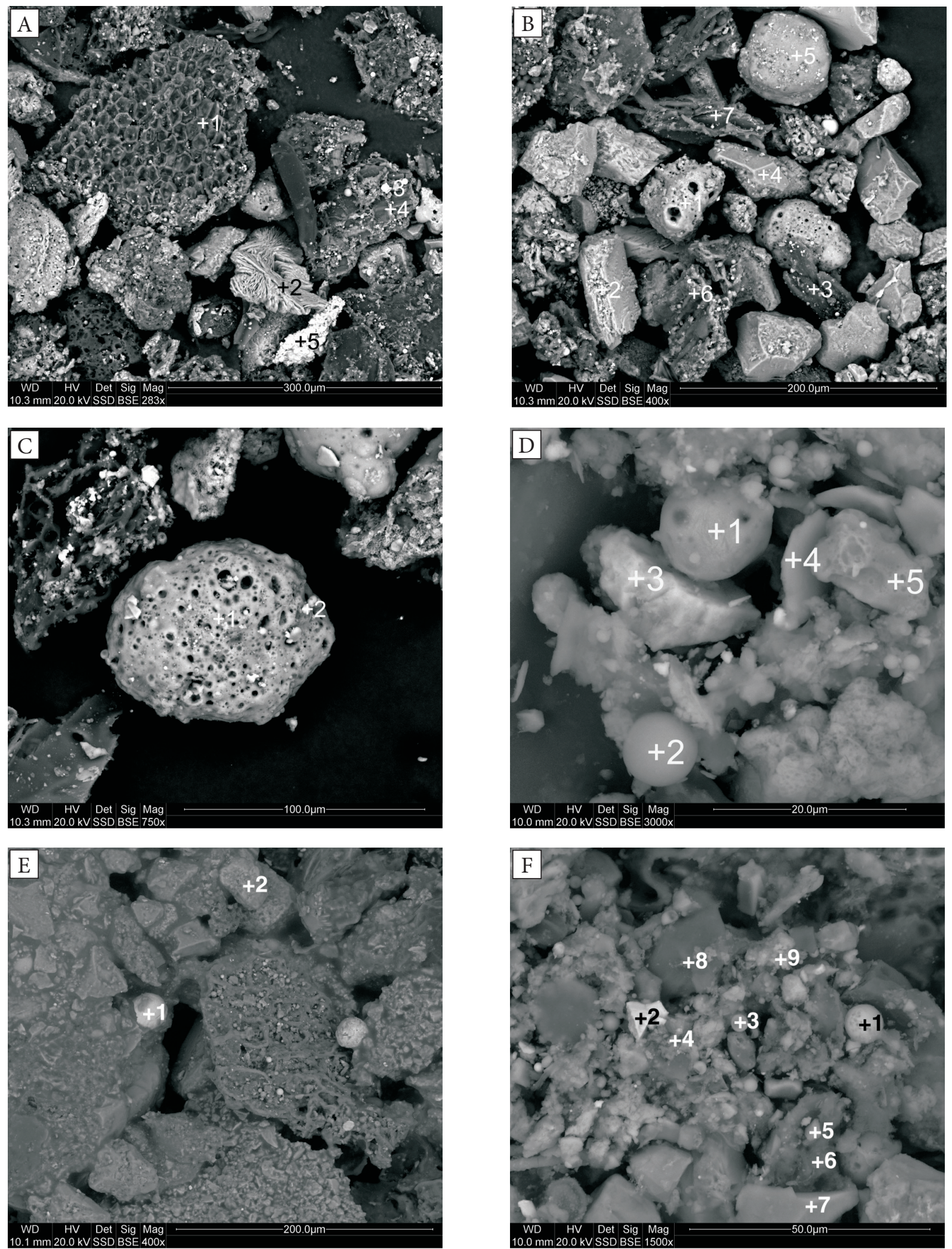

Fig. 3. SEM microphotographs of the deposited particulate matter (PM ): A) PM sample 1: 1 and 4 - organic fragments, 2 - gypsum, 3 - iron oxide, 5 - pieces of iron; B) PM sample 2: 1 - pieces of aluminosilicate glass, 2 - hydrophilite, 3 - organic pieces, 4 - quartz, 5 - feldspar, 6 and 7 - weathered plagioclase; C) PM sample 2: 1 and 2 -aluminosilicate glass; D) PM sample 5: 1 and 2 - fly ash, 3 - iron oxide, 4 - plagioclase, 5 - silicate glass; E) PM sample 6: 1 - iron oxide, 2 - feldspar; F) PM sample 7: 1 - spherical carbonate form, 2 - iron oxide, 3 - fly ash, 4 and 6 - a piece of aluminosilicate glass, 5 and 7 - quartz, 8 and 9 - plagioclase 
Table 1

Concentrations of selected elements in the deposited particulate matter (PM)

\begin{tabular}{|c|c|c|c|c|}
\hline \multirow{3}{*}{ Element } & \multicolumn{2}{|c|}{ PM sample - point 1} & \multicolumn{2}{|c|}{ PM sample - point 7} \\
\hline & concentration & SD & concentration & SD \\
\hline & \multicolumn{2}{|c|}{ mg/kg d.m. } & \multicolumn{2}{|c|}{ mg/kg d.m. } \\
\hline $\mathrm{Al}$ & 6300 & 199 & 6400 & 248.4 \\
\hline As & 8.3 & 0.76 & 21 & 7.48 \\
\hline $\mathrm{Ba}$ & 134 & 2.35 & 89 & 1.55 \\
\hline $\mathrm{Be}$ & 0.63 & 0.02 & 0.45 & 0.05 \\
\hline $\mathrm{Ca}$ & 19400 & 248 & 64400 & 596.1 \\
\hline $\mathrm{Cd}$ & 1.1 & 0.11 & 1.2 & 0.25 \\
\hline Co & 2.6 & 0.34 & 2.1 & 0.578 \\
\hline $\mathrm{Cr}$ & 68 & 0.25 & 112 & 0.29 \\
\hline $\mathrm{Cu}$ & 64 & 1.20 & 185 & 8.24 \\
\hline $\mathrm{Fe}$ & 44700 & 520 & 36200 & 228 \\
\hline $\mathrm{Ga}$ & 4.8 & 0.45 & 3.7 & 0.99 \\
\hline $\mathrm{K}$ & 3620 & 37.5 & 4080 & 18.6 \\
\hline $\mathrm{Li}$ & 19.5 & 0.20 & 22.7 & 0.31 \\
\hline $\mathrm{Mg}$ & 8000 & 88.4 & 9070 & 57.0 \\
\hline $\mathrm{Mn}$ & 603 & 9.19 & 644 & 4.79 \\
\hline $\mathrm{Na}$ & 1050 & 11.4 & 1010 & 11.8 \\
\hline $\mathrm{Ni}$ & 25.8 & 0.72 & 23.1 & 0.90 \\
\hline $\mathrm{Pb}$ & 83 & 1.06 & 88 & 1.86 \\
\hline $\mathrm{Rb}$ & 6.8 & 0.12 & 6.4 & 0.87 \\
\hline $\mathrm{Si}$ & 600 & 4.20 & 1438 & 5.73 \\
\hline Sn & 11 & 1.61 & 14 & 2.08 \\
\hline $\mathrm{Sr}$ & 169 & 2.34 & 112 & 0.95 \\
\hline $\mathrm{Ti}$ & 194 & 7.05 & 252 & 3.84 \\
\hline $\mathrm{V}$ & 24 & 0.35 & 36 & 0.22 \\
\hline $\mathrm{Y}$ & 2.23 & 0.04 & 1.8 & 0.10 \\
\hline $\mathrm{Zn}$ & 1010 & 10.3 & 10630 & 36.7 \\
\hline $\mathrm{Zr}$ & 4.4 & 0.65 & 4.7 & 0.20 \\
\hline
\end{tabular}

SD - standard deviation; d.m. - dry mass.

Daily intakes (EDI) estimated for resuspended PM have shown that the highest values were observed for ingestion exposure pathway than for dermal contact, and finally for inhalation, both for children and adults, as well as, for carcinogenic and non-carcinogenic elements (Tab. 2). The hazard quotient (HQ) values calculated for all three exposure pathways were $<1$, which indicated the lack of essential non-carcinogenic risk levels for particular elements (Tab. 3). The non-carcinogenic risk decreased for children and adults, in each exposure pathway, in the following order: $\mathrm{Fe}>\mathrm{Al}>\mathrm{Zn}>\mathrm{Mn}$ $>\mathrm{Sr}>\mathrm{Cu}>\mathrm{Ba}>\mathrm{Cr}>\mathrm{Pb}>\mathrm{V}>\mathrm{Ni}>\mathrm{Li}>\mathrm{As}>\mathrm{Sn}>$ $\mathrm{Zr}>\mathrm{Co}>\mathrm{Cd}>\mathrm{Be}$. The hazard index values calculated in each pathway for all elements $\left(\mathrm{HI}_{\text {elem }}\right)$ were also $<1$ (Tab. 3), which indicated the lack of essential health risk levels as well. The mean hazard index values (as arithmetic mean from points 1 and 7) for all elements in each pathway $\left(\mathrm{HI}_{\text {elem }}\right)$ were as follows: in ingestion for children 3.55E-01, for adults 3.80E-02; in dermal contact for children 9.90E-04, for adults 1.52E-04; in inhalation of resuspended PM for children 9.89E-06, for adults 5.59E-06. Also, the hazard index values for every single element in all three pathways $\left(\mathrm{HI}_{\text {path }}\right)$ were $<1$, both for children and adults (Tab. 4).

The estimated $\mathrm{R}$ values for As and $\mathrm{Cr}$, which were considered as carcinogenic elements, in single exposure pathways were below the level of 1.00E-05, which indicated the lack of essential carcinogenic risk (Tab. 3), as well as, the $\mathrm{R}$ values for both carcinogenic elements. 


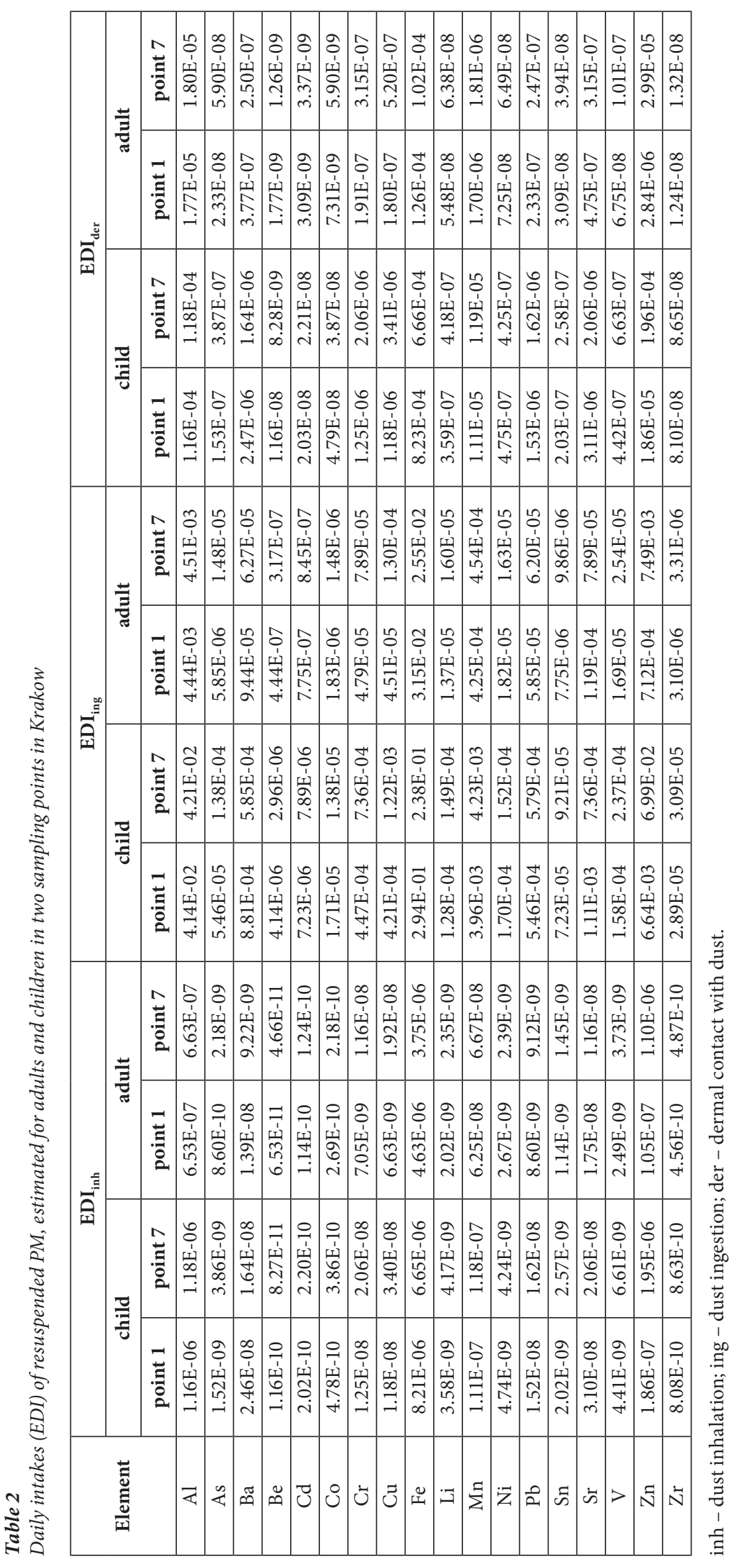




\begin{tabular}{|c|c|c|c|c|c|c|c|c|c|c|c|c|c|c|c|c|c|c|c|c|c|c|c|c|c|c|}
\hline \multirow{4}{*}{ 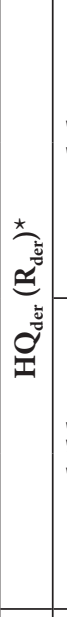 } & & 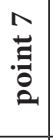 & 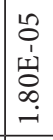 & \begin{tabular}{|c}
$\infty$ \\
0 \\
1 \\
1 \\
0 \\
0 \\
1 \\
1
\end{tabular} & $\begin{array}{l}\infty \\
0 \\
0 \\
1 \\
1 \\
\infty \\
\infty \\
\infty \\
\infty \\
\infty\end{array}$ & 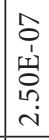 & 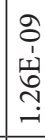 & 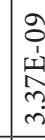 & $\mid \begin{array}{l}q \\
c \\
b \\
\sigma \\
\sigma\end{array}$ & & 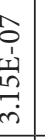 & 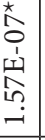 & 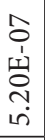 & 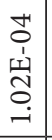 & $\mid \begin{array}{c}\infty \\
0 \\
1 \\
1 \\
\infty \\
0 \\
0 \\
b\end{array}$ & 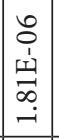 & 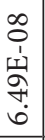 & 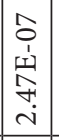 & \begin{tabular}{l}
$\infty$ \\
0 \\
0 \\
1 \\
\multirow{1}{1}{} \\
$\dot{m}$
\end{tabular} & 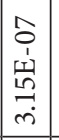 & $\begin{array}{l}0 \\
0 \\
1 \\
\vdots \\
\vdots \\
-i\end{array}$ & 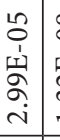 & 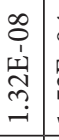 & & 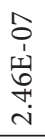 & 节 \\
\hline & 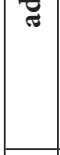 & $\begin{array}{l}\overrightarrow{.} \\
\vec{z} \\
\vdots \\
\vdots\end{array}$ & 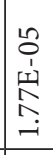 & \begin{tabular}{|c|c}
$\infty$ \\
0 \\
1 \\
\\
$\tilde{n}$ \\
$i$
\end{tabular} & 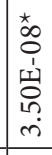 & 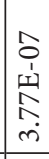 & 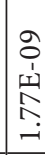 & 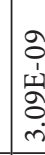 & $\mid \begin{array}{c}g \\
c \\
\frac{1}{m} \\
\end{array}$ & & 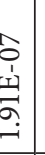 & 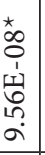 & $\begin{array}{l}\hat{o} \\
1 \\
1 \\
\text { d } \\
\infty \\
- \\
\end{array}$ & 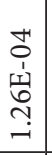 & $\mid \begin{array}{c}\infty \\
0 \\
1 \\
1 \\
\infty \\
+1 \\
+1 \\
i n\end{array}$ & 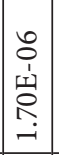 & 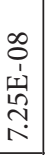 & 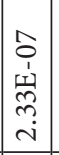 & $\begin{array}{l}\infty \\
0 \\
1 \\
\omega \\
2 \\
0 \\
\dot{n}\end{array}$ & 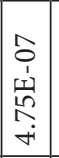 & 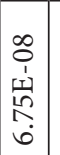 & $\begin{array}{l}8 \\
0 \\
1 \\
1 \\
+ \\
0 \\
i\end{array}$ & 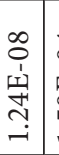 & & $\begin{array}{l}\text { ô } \\
\text { İ } \\
\vec{m} \\
\text { m. }\end{array}$ & \\
\hline & $\bar{\theta}$ & 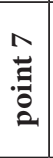 & 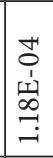 & \begin{tabular}{|c|c}
$\hat{o}$ \\
1 \\
1 \\
1 \\
0 \\
$\dot{n}$ \\
\end{tabular} & 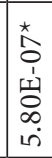 & 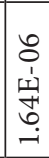 & 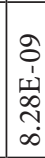 & 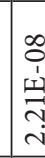 & 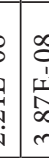 & & 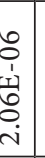 & 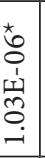 & 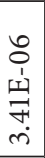 & 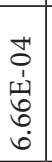 & 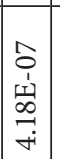 & 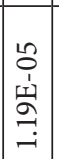 & 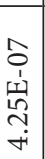 & 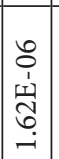 & 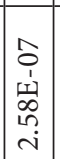 & $\left|\begin{array}{c}0 \\
0 \\
1 \\
1 \\
0 \\
0 \\
i\end{array}\right|$ & $\begin{array}{l}0 \\
0 \\
1 \\
1 \\
0 \\
0 \\
\dot{0}\end{array}$ & $\begin{array}{l}+ \\
0 \\
1 \\
1 \\
0 \\
-3 \\
-1\end{array}$ & $\begin{array}{l}\infty \\
0 \\
\vdots \\
1 \\
\vdots \\
0 \\
\infty \\
\infty\end{array}$ & $\begin{array}{l}0 \\
0 \\
1 \\
\vdots \\
0 \\
- \\
-1\end{array}$ & 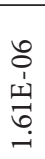 & \\
\hline & & 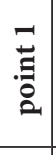 & 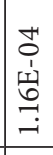 & \begin{tabular}{|c}
0 \\
0 \\
1 \\
1 \\
\\
\\
$-i$
\end{tabular} & 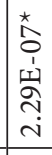 & 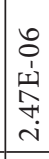 & 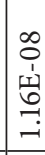 & 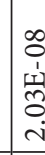 & 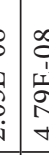 & & 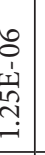 & 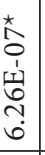 & 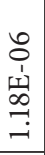 & 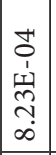 & 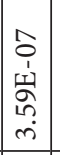 & $\begin{array}{l}2 \\
0 \\
0 \\
\omega \\
\exists \\
ت \\
\end{array}$ & 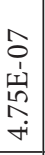 & $\begin{array}{c}0 \\
0 \\
1 \\
\omega \\
\tilde{n} \\
\\
-1\end{array}$ & 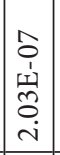 & 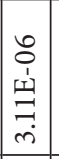 & $\begin{array}{l}\hat{0} \\
1 \\
1 \\
y \\
f \\
\dot{+} \\
\end{array}$ & $\begin{array}{l}2 \\
0 \\
1 \\
1 \\
0 \\
\infty \\
-1\end{array}$ & $\begin{array}{l}0 \\
0 \\
1 \\
1 \\
0 \\
0 \\
\infty\end{array}$ & & $\begin{array}{l}\hat{o} \\
\text { 1 } \\
\text { In } \\
\text { hn } \\
\infty\end{array}$ & \\
\hline & & 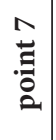 & 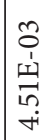 & 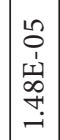 & 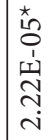 & 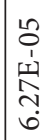 & 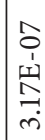 & $\begin{array}{c}a \\
0 \\
1 \\
1 \\
1 \\
\infty \\
\infty\end{array}$ & 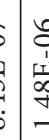 & & 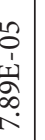 & 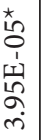 & 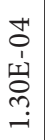 & 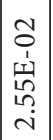 & 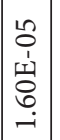 & 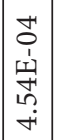 & 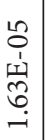 & 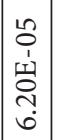 & $\begin{array}{l}0 \\
0 \\
1 \\
1 \\
0 \\
0 \\
o \\
\sigma\end{array}$ & 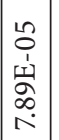 & 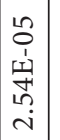 & 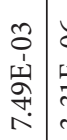 & $\begin{array}{l}0 \\
⿱ \\
1 \\
m \\
m \\
m \\
m\end{array}$ & 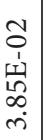 & 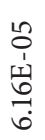 & $\bar{v}$ \\
\hline & & $\vec{\Xi}$ & 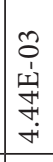 & \begin{tabular}{|c|c}
0 \\
1 \\
1 \\
1 \\
0 \\
$\infty$ \\
$i n$ \\
\end{tabular} & 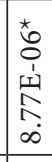 & 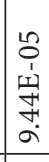 & 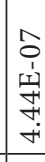 & $\begin{array}{l}\hat{a} \\
\hat{1} \\
\\
\\
\end{array}$ & 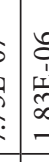 & & 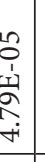 & 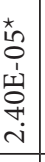 & 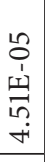 & 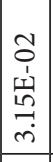 & 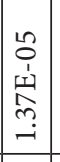 & 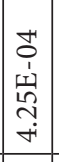 & $\begin{array}{c}n \\
0 \\
1 \\
1 \\
\vdots \\
o \\
- \\
\end{array}$ & 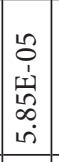 & 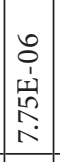 & 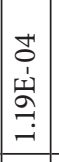 & $\begin{array}{l}2 \\
0 \\
1 \\
1 \\
\vdots \\
- \\
-1\end{array}$ & 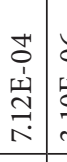 & 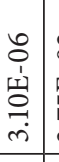 & 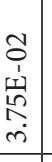 & 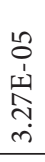 & \\
\hline & & 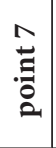 & 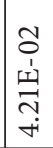 & 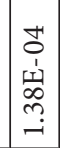 & 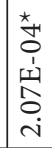 & 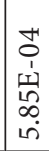 & 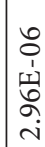 & 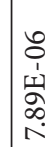 & 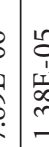 & & 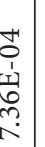 & 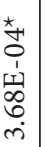 & 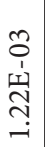 & $\left|\begin{array}{c}0 \\
1 \\
\hat{\omega} \\
\tilde{N} \\
\vec{i}\end{array}\right|$ & 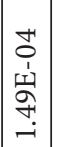 & $\left|\begin{array}{c}0 \\
0 \\
\omega \\
\tilde{N} \\
\sim \\
+ \\
+\end{array}\right|$ & 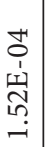 & $\mid \begin{array}{c}0 \\
0 \\
1 \\
\\
\hat{2} \\
i \\
i n\end{array}$ & 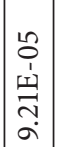 & $\begin{array}{l}T \\
0 \\
1 \\
1 \\
0 \\
0 \\
\end{array}$ & 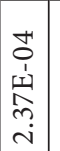 & 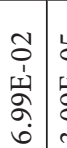 & $\begin{array}{l}2 \\
0 \\
1 \\
\vdots \\
\dot{n} \\
\dot{m}\end{array}$ & $\begin{array}{l}\overrightarrow{0} \\
1 \\
\underline{2} \\
\hat{n} \\
0 \\
0\end{array}$ & 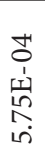 & \\
\hline & & $\begin{array}{l}\vec{Z} \\
\vec{z} \\
\vdots \\
\vdots\end{array}$ & 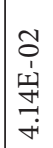 & $\begin{array}{l}n \\
0 \\
1 \\
1 \\
0 \\
0 \\
1 \\
n \\
n\end{array}$ & 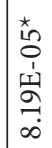 & $\begin{array}{l}\vec{t} \\
0 \\
1 \\
\omega \\
0 \\
0 \\
\infty \\
0\end{array}$ & 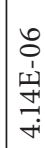 & $\begin{array}{l}0 \\
0 \\
1 \\
\\
\\
\end{array}$ & 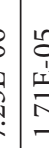 & & 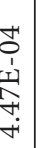 & 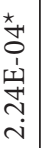 & 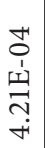 & $\mid \begin{array}{c}\overrightarrow{0} \\
\hat{1} \\
\hat{y} \\
2 \\
\dot{i}\end{array}$ & 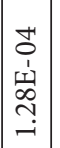 & $\left|\begin{array}{c}0 \\
0 \\
1 \\
1 \\
0 \\
2 \\
\dot{m}\end{array}\right|$ & 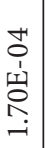 & 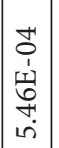 & 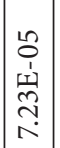 & 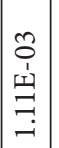 & 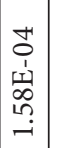 & $\begin{array}{l}0 \\
0 \\
+ \\
1 \\
+ \\
0 \\
0 \\
0\end{array}$ & \begin{tabular}{l}
20 \\
+ \\
1 \\
\multicolumn{2}{c}{} \\
0 \\
$i$
\end{tabular} & 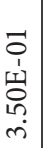 & 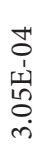 & \\
\hline & \multirow{2}{*}{$\underset{\vec{\Xi}}{\vec{\Xi}}$} & 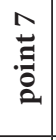 & 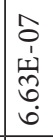 & 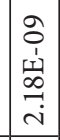 & 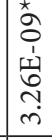 & 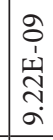 & 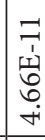 & 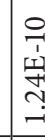 & 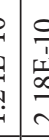 & & 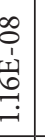 & 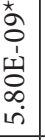 & 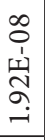 & $\begin{array}{c}0 \\
0 \\
1 \\
n \\
1 \\
r \\
\\
\end{array}$ & 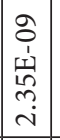 & $\mid \begin{array}{l}\infty \\
0 \\
1 \\
1 \\
\hat{1} \\
0 \\
0 \\
0\end{array}$ & 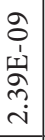 & 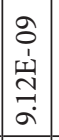 & 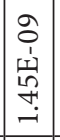 & \begin{tabular}{c}
$\infty$ \\
0 \\
1 \\
1 \\
0 \\
\hdashline \\
-1 \\
-1
\end{tabular} & $\begin{array}{c}0 \\
0 \\
1 \\
1 \\
\tilde{N} \\
\hat{n}\end{array}$ & 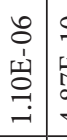 & 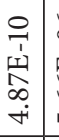 & 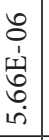 & 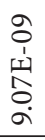 & \\
\hline & & $\begin{array}{l}\vec{z} \\
\vec{z} \\
\vdots\end{array}$ & $\begin{array}{l}\hat{0} \\
\hat{1} \\
\omega \\
\hat{n} \\
\hat{n} \\
b\end{array}$ & $\begin{array}{c}0 \\
1 \\
1 \\
0 \\
0 \\
0 \\
\infty \\
\infty\end{array}$ & 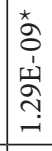 & 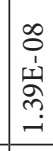 & 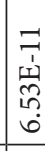 & 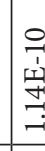 & 4 & & 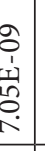 & 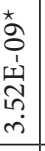 & $\begin{array}{l}a \\
\hat{1} \\
\hat{1} \\
\hat{0} \\
\dot{b}\end{array}$ & $\begin{array}{c}0 \\
0 \\
1 \\
\hat{n} \\
6 \\
+ \\
+\end{array}$ & $\left|\begin{array}{c}0 \\
0 \\
i \\
\tilde{N} \\
0 \\
i \\
i\end{array}\right|$ & 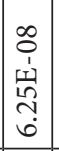 & $\begin{array}{l}0 \\
0 \\
1 \\
1 \\
0 \\
\text { in } \\
i\end{array}$ & $\mid \begin{array}{c}0 \\
0 \\
1 \\
1 \\
0 \\
0 \\
\infty \\
\infty\end{array}$ & 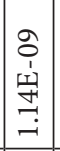 & 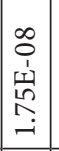 & 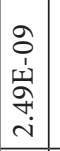 & $\begin{array}{l}0 \\
0 \\
0 \\
4 \\
0 \\
-1\end{array}$ & 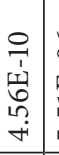 & 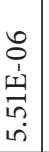 & 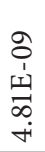 & \\
\hline & \multirow[t]{2}{*}{ 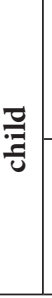 } & 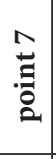 & $\begin{array}{l}0 \\
0 \\
1 \\
0 \\
0 \\
= \\
-1\end{array}$ & $\begin{array}{l}a \\
o \\
1 \\
1 \\
0 \\
\infty \\
r \\
\dot{n}\end{array}$ & 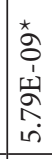 & 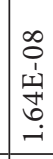 & $\begin{array}{l}7 \\
1 \\
\mathbf{1} \\
N \\
\infty \\
\infty\end{array}$ & 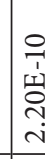 & 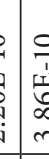 & 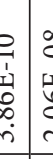 & 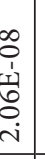 & 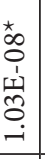 & $\begin{array}{l}0 \\
0 \\
+ \\
1 \\
0 \\
+1 \\
\end{array}$ & $\begin{array}{l}0 \\
0 \\
1 \\
\hat{1} \\
0 \\
0 \\
0\end{array}$ & 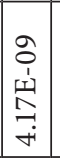 & 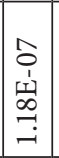 & 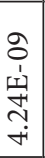 & 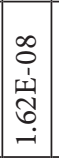 & 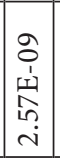 & $\left|\begin{array}{c}0 \\
0 \\
1 \\
1 \\
0 \\
0 \\
i\end{array}\right|$ & $\begin{array}{l}0 \\
0 \\
1 \\
1 \\
\vec{b} \\
\vdots \\
\vdots\end{array} \mid$ & 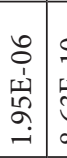 & 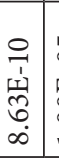 & 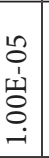 & 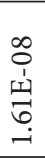 & \\
\hline & & $\begin{array}{c}\vec{z} \\
\vec{a} \\
\vdots \\
\vdots\end{array}$ & 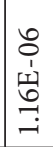 & 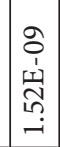 & 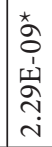 & $\begin{array}{l}\infty \\
0 \\
0 \\
1 \\
\text { t } \\
+1 \\
\text { in }\end{array}$ & 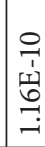 & 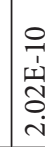 & 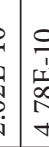 & & 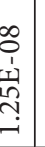 & 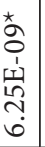 & \begin{tabular}{l}
$\infty$ \\
0 \\
1 \\
1 \\
$\infty$ \\
\hdashline \\
$ت$ \\
$ت$
\end{tabular} & 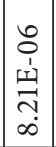 & $\left|\begin{array}{c}2 \\
0 \\
1 \\
1 \\
0 \\
0 \\
n \\
n\end{array}\right|$ & 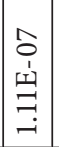 & 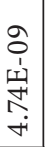 & 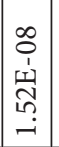 & 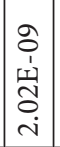 & 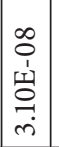 & $\begin{array}{c}9 \\
\hat{1} \\
1 \\
\vec{F} \\
\dot{+}\end{array}$ & 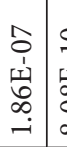 & $\begin{array}{l}0 \\
1 \\
1 \\
0 \\
0 \\
0 \\
\infty \\
\infty\end{array}$ & 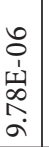 & 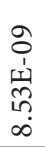 & \\
\hline & & $\frac{\pi}{\pi}$ & $\stackrel{\pi}{\exists}$ & & 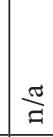 & ב็ & $\stackrel{\pi}{z}$ & 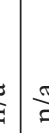 & 㫌 & 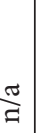 & in & $\frac{\pi}{a}$ & 亲 & $\frac{\pi}{a}$ & $\frac{\pi}{a}$ & $\stackrel{\pi}{\Xi}$ & $\frac{\pi}{a}$ & $\stackrel{\pi}{a}$ & $\frac{\pi}{a}$ & $\frac{\pi}{a}$ & $\frac{\pi}{a}$ & $\stackrel{\pi}{\beth}$ & $\stackrel{\pi}{\Xi}$ & $\stackrel{\pi}{z}$ & \\
\hline & $\overline{\widetilde{Z}}$ & & & $\begin{array}{c}-t \\
0 \\
1 \\
\dot{1} \\
0 \\
\dot{m} \\
\end{array}$ & $\int_{3}^{\frac{z}{3}}$ & 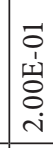 & 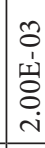 & 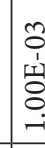 & 方 & 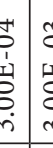 & & $\stackrel{\pi}{\Xi}$ & $\underset{+}{+}$ & 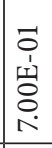 & $\begin{array}{c}0 \\
0 \\
1 \\
\dot{1} \\
0 \\
i \\
i\end{array}$ & $\begin{array}{r}0 \\
0 \\
1 \\
0 \\
+ \\
+ \\
-1 \\
\end{array}$ & $\begin{array}{l}\mathbf{0} \\
\text { 1 } \\
\vdots \\
0 \\
i \\
\end{array}$ & 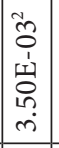 & $\begin{array}{l}\overrightarrow{0} \\
\hat{1} \\
\hat{\omega} \\
0 \\
\dot{0} \\
\end{array}$ & $\begin{array}{c}0 \\
0 \\
1 \\
\dot{1} \\
0 \\
0 \\
\dot{0} \\
\end{array}$ & $\begin{array}{l}m \\
0 \\
1 \\
\dot{0} \\
0 \\
\dot{1} \\
\end{array}$ & $\begin{array}{l}\overrightarrow{0} \\
+ \\
\dot{1} \\
\vdots \\
\dot{r} \\
\dot{r}\end{array}$ & $\begin{array}{l}2 \\
0 \\
1 \\
1 \\
0 \\
0 \\
\infty\end{array}$ & $\stackrel{\pi}{\exists}$ & $\stackrel{\pi}{z}$ & \\
\hline & 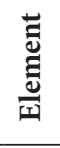 & & 飞 & 里 & 告 & & m & 0 & $s$ & 8 & $u$ & u & $\tilde{J}$ & tक & $ت$ & $\sum_{\Sigma}^{\Xi}$ & Z & $\overrightarrow{2}$ & מี & $\dot{\omega}$ & $>$ & งี & $\dot{N}$ & \pm & $x^{0}$ & \\
\hline
\end{tabular}


Table 4

Total health risk from exposure to resuspended PM in Kraków

\begin{tabular}{|c|c|c|c|c|}
\hline \multirow{3}{*}{ Element } & \multicolumn{4}{|c|}{$\mathrm{HI}_{\text {path }}\left(\mathrm{R}_{\text {path }}\right)^{\star}$ for three exposure pathways } \\
\hline & \multicolumn{2}{|c|}{ child } & \multicolumn{2}{|c|}{ adult } \\
\hline & point 1 & point 7 & point 1 & point 7 \\
\hline $\mathrm{Al}$ & $4.15 \mathrm{E}-02$ & $4.22 \mathrm{E}-02$ & $4.46 \mathrm{E}-03$ & $4.53 \mathrm{E}-03$ \\
\hline As & $5.47 \mathrm{E}-05$ & $1.38 \mathrm{E}-04$ & $5.87 \mathrm{E}-06$ & $1.49 \mathrm{E}-05$ \\
\hline $\mathrm{As}_{\text {canc }}{ }^{*}$ & $8.21 \mathrm{E}-05$ & $2.08 \mathrm{E}-04$ & $8.81 \mathrm{E}-06$ & $2.23 \mathrm{E}-05$ \\
\hline $\mathrm{Ba}$ & $8.84 \mathrm{E}-04$ & $5.87 \mathrm{E}-04$ & $9.48 \mathrm{E}-05$ & $6.30 \mathrm{E}-05$ \\
\hline $\mathrm{Be}$ & $4.15 \mathrm{E}-06$ & $2.97 \mathrm{E}-06$ & $4.46 \mathrm{E}-07$ & $3.18 \mathrm{E}-07$ \\
\hline $\mathrm{Cd}$ & $7.25 \mathrm{E}-06$ & 7.91E-06 & $7.78 \mathrm{E}-07$ & $8.49 \mathrm{E}-07$ \\
\hline Co & $1.71 \mathrm{E}-05$ & $1.38 \mathrm{E}-05$ & $1.84 \mathrm{E}-06$ & $1.49 \mathrm{E}-06$ \\
\hline $\mathrm{Cr}$ & $4.48 \mathrm{E}-04$ & 7.39E-04 & $4.81 \mathrm{E}-05$ & $7.92 \mathrm{E}-05$ \\
\hline $\mathrm{Cr}_{\text {canc }}{ }^{*}$ & $2.24 \mathrm{E}-04$ & $3.69 \mathrm{E}-04$ & $2.41 \mathrm{E}-05$ & $3.96 \mathrm{E}-05$ \\
\hline $\mathrm{Cu}$ & $4.22 \mathrm{E}-04$ & $1.22 \mathrm{E}-03$ & $4.53 \mathrm{E}-05$ & $1.31 \mathrm{E}-04$ \\
\hline $\mathrm{Fe}$ & $2.95 \mathrm{E}-01$ & $2.39 \mathrm{E}-01$ & $3.16 \mathrm{E}-02$ & $2.56 \mathrm{E}-02$ \\
\hline $\mathrm{Li}$ & $1.29 \mathrm{E}-04$ & $1.50 \mathrm{E}-04$ & $1.38 \mathrm{E}-05$ & $1.61 \mathrm{E}-05$ \\
\hline $\mathrm{Mn}$ & $3.98 \mathrm{E}-03$ & $4.25 \mathrm{E}-03$ & $4.27 \mathrm{E}-04$ & $4.56 \mathrm{E}-04$ \\
\hline $\mathrm{Ni}$ & $1.70 \mathrm{E}-04$ & $1.52 \mathrm{E}-04$ & $1.83 \mathrm{E}-05$ & $1.63 \mathrm{E}-05$ \\
\hline $\mathrm{Pb}$ & $5.47 \mathrm{E}-04$ & $5.80 \mathrm{E}-04$ & $5.87 \mathrm{E}-05$ & $6.23 \mathrm{E}-05$ \\
\hline Sn & $7.25 \mathrm{E}-05$ & $9.23 \mathrm{E}-05$ & 7.78E-06 & $9.90 \mathrm{E}-06$ \\
\hline $\mathrm{Sr}$ & $1.11 \mathrm{E}-03$ & 7.39E-04 & $1.20 \mathrm{E}-04$ & 7.92E-05 \\
\hline $\mathrm{V}$ & $1.58 \mathrm{E}-04$ & $2.37 \mathrm{E}-04$ & $1.70 \mathrm{E}-05$ & $2.55 \mathrm{E}-05$ \\
\hline $\mathrm{Zn}$ & $6.66 \mathrm{E}-03$ & 7.01E-02 & $7.14 \mathrm{E}-04$ & $7.52 \mathrm{E}-03$ \\
\hline $\mathrm{Zr}$ & $2.90 \mathrm{E}-05$ & $3.10 \mathrm{E}-05$ & $3.11 \mathrm{E}-06$ & $3.32 \mathrm{E}-06$ \\
\hline $\mathrm{HI}_{\text {total }}$ & $3.51 \mathrm{E}-01$ & $3.60 \mathrm{E}-01$ & $3.77 \mathrm{E}-02$ & $3.86 \mathrm{E}-02$ \\
\hline $\mathrm{R}_{\text {total }}{ }^{*}$ & $3.06 \mathrm{E}-04$ & $5.77 \mathrm{E}-04$ & $3.29 \mathrm{E}-05$ & $6.19 \mathrm{E}-05$ \\
\hline
\end{tabular}

path - pathway; ${ }^{*}$ carcinogenic risk for $\mathrm{As}_{\text {canc }}$ and $\mathrm{Cr}_{\text {canc }} ; \mathrm{HI}_{\text {total }}$ - total risk for non-carcinogenic elements in deposited $\mathrm{PM}$; $\mathrm{R}_{\text {total }}$ - total risk for carcinogenic risk in deposited PM.

The mean risk values (as arithmetic mean from points 1 and 7) for both elements in each exposure pathway $\left(\mathrm{R}_{\text {elem }}\right)$ were as follows: in ingestion for children 4.40E-04, for adults 4.72E-05; in dermal contact for children 1.23E-06, for adults $1.88 \mathrm{E}-07$; in inhalation for children $1.23 \mathrm{E}-08$, for adults 6.49E-09. The carcinogenic risk values in all three exposure pathways $\left(\mathrm{R}_{\mathrm{path}}\right)$, both for As and $\mathrm{Cr}$, were higher than acceptable level of $1.00 \mathrm{E}-05$, both for children and adults in case of $\mathrm{Cr}$, and for children in case of As (Tab. 4).

The total non-carcinogenic risk values in all three exposure pathways for all elements $\left(\mathrm{HI}_{\text {total }}\right)$ were $<1$, however in case of children the total HI value between 1.00E-01 and $1.00 \mathrm{E}+00$ (Tab. 4) pointed the low risk level (Lemly 1996). The total carcinogenic risk values in all three exposure pathways $\left(\mathrm{R}_{\text {total }}\right)$ for both carcinogenic elements exceeded the acceptable level of 1.00E-05, both for children and adults.

The health risk assessed in the study includes only exposure to resuspended PM. We have to bear in mind that concerning such risks, the inhaled air exposure pathway is the first to be assessed (Gruszecka-Kosowska 2016). As for the carcinogenic elements, only As and $\mathrm{Cr}$ were analysed because there were available SF values in toxicological databases. Also, not all of analysed non-carcinogenic elements had RfD values in toxicological databases. Thus, the entire risk value, both carcinogenic and non-carcinogenic, will be higher than those estimated in our calculation. There is a probability that these real risk values will significantly exceed the acceptable human health risk levels. 


\section{CONCLUSIONS}

From the study the following conclusions were derived:

1. Calculated in winter months 2015-2016, the level of PM deposition amounted to $0.0646 \mathrm{~g} / \mathrm{m}^{2}$ per day in the sampling point located in the centre of Krakow and $0.0328 \mathrm{~g} / \mathrm{m}^{2}$ in the sampling point located in the northern district of the city. PM deposition did not exceed the allowed daily dust deposit level, equal to $0.547 \mathrm{~g} / \mathrm{m}^{2}$ per day.

2. The mineralogical analysis showed that quartz, calcite, potassium feldspar, plagioclase, kaolinite, and gypsum were the main mineralogical components of the deposited PM. Only in few samples illite, dolomite, and apatite were observed. In case of two sampling points, portlandite and gismondine as well as chlorite and hematite were found.

3. Considering trace elements in the deposited $\mathrm{PM}$, very high concentrations were stated for $\mathrm{Ca}, \mathrm{Fe}, \mathrm{Mg}, \mathrm{Al}, \mathrm{K}, \mathrm{Si}, \mathrm{Na}$, and $\mathrm{Mn}$, and high concentrations for $\mathrm{Ti}, \mathrm{Cu}, \mathrm{Zn}, \mathrm{As}, \mathrm{Pb}, \mathrm{Cr}, \mathrm{Ti}, \mathrm{V}$, $\mathrm{Li}, \mathrm{Sr}$, and $\mathrm{Ni}$.

4. The highest estimated daily intakes (EDI) for resuspended PM, were stated for the ingestion exposure pathway than for dermal contact, and finally for inhalation, both for children and adults as well as for carcinogenic and noncarcinogenic elements.

5. The mean hazard quotient (HQ) values for all of the three exposure pathways decreased in the following order: $\mathrm{Fe}>\mathrm{Al}>\mathrm{Zn}>\mathrm{Mn}>\mathrm{Sr}>$ $\mathrm{Cu}>\mathrm{Ba}>\mathrm{Cr}>\mathrm{Pb}>\mathrm{V}>\mathrm{Ni}>\mathrm{Li}>\mathrm{As}>\mathrm{Sn}>\mathrm{Zr}$ $>\mathrm{Co}>\mathrm{Cd}>\mathrm{Be}$, both for children and adults.

6. The hazard index values for all elements in each exposure pathway $\left(\mathrm{HI}_{\text {elem }}\right)$ and for single elements in all exposure pathways $\left(\mathrm{HI}_{\text {path }}\right)$ were $<1$, both for children and adults.

7. The carcinogenic risk values in each exposure pathway $\left(\mathrm{R}_{\text {elem }}\right)$ did not exceed the acceptable level of 1.00E-05 for As and Cr. However, the risk values from all three exposure pathways $\left(R_{\text {path }}\right)$ were unacceptable in case of $\mathrm{Cr}$ both for children and adults, and in the case of As for children.

8. The total non-carcinogenic risk $\left(\mathrm{HI}_{\text {total }}\right)$ values in all three exposure pathways for all elements were $<1$, however in case of children the risk value pointed the low risk level.

9. The total carcinogenic risk $\left(\mathrm{R}_{\text {total }}\right)$ values in all three exposure pathways for As and $\mathrm{Cr}$ exceeded the acceptable level, both for children and adults.

10. The research indicated that the mineral and chemical composition of PM can be harmful for Krakow's inhabitants.

\section{REFERENCES}

Aleksandropoulou V. \& Lazaridis M., 2013. Development and application of a model (ExDoM) for calculating the respiratory tract dose and retention of particles under variable exposure conditions. Air Quality, Atmosphere \& Health, 6, 1, 13-26.

ATSDR, 2005. Public health assessment guidance manual. Agency for Toxic Substances and Disease Registry

Baldacci S., Maio S., Cerrai S., Sarno G., Baïzb N., Simoni M., Annesi-Maesano I. \& Viegi G., 2015. Allergy and asthma: Effects of the exposure to particulate matter and biological allergens. Respiratory Medicine, 109, 1089-1104.

Beig G., Chate D.M., Ghude S.D., Mahajan A.S., Srinivas R., Ali K., Sahu S.K., Parkhi N., Surendran D. \& Trimbake H.R., 2013. Quantifying the effect of air quality control measures during the 2010 Commonwealth Games at Delhi, India. Atmospheric Environment, 80, 455-463.

Brook R.D., Franklin B., Cascio W., Hong Y., Howard G., Lipsett M., Luepker R., Mittleman M., Samet J., Smith S.C. \& Tager I., 2004. Air pollution and cardiovascular disease. Circulation, 109, 2655-2671.

Brunekreef B. \& Holgate S.T., 2002. Air pollution and health. Lancet, 360, 1233-1242.

EEA, 2014. Air Quality in Europe. Report No 5/2014.

Fereira-Baptista L. \& De Miguel E., 2005. Geochemistry and risk assessment of street dust in Luanda, Angola: A tropical urban environment. Atmospheric Environment, 39, 4501-4512.

Fuzzi S., Baltensperger U., Carslaw K. et al., 2015. Particulate matter, air quality and climate: lessons learned and future needs. Atmospheric Chemistry and Physics, 15, 8217-8299.

Götschi T., Heinrich J., Sunyer K. \& Künzli N., 2008. Longterm effects of ambient air pollution on lung function. A review. Epidemiology, 19, 690-701.

Grantz D.A., Garner J.H.B. \& Johnson D.W., 2003. Ecological effects of particulate matter. Environment International, 29, 213-239.

Gruszecka-Kosowska A., 2016. Assessment of the Kraków inhabitants' health risk caused by the exposure to inhalation of outdoor air contaminants. Stochastic Environmental Research and Risk Assessment, doi:10.1007/ s00477-016-1366-8.

Guarnieri M. \& Balmes J.R., 2014. Outdoor air pollution and asthma. Lancet, 383, 1581-1592.

Hansen L.D., Silberman D. \& Fisher G.L., 1981. Crystalline components of stack collected, size-fractionated coal fly ash. Environmental Science \& Technology, 15, 1057-1062. 
Hulett L.D., Weinberger A.J., Northcutt K.J. \& Ferguson M., 1980. Chemical species in fly ash from coal-burning power plant. Science, 210, 1356-1358.

IPCC, 2007. Climate Change 2007: The Physical Science Basis. Contribution of Working Group I to the Fourth Assessment Report of the IPCC, Cambridge University Press.

Jiming H.A.O., Kebin H.E., Duan L., Junhua L. \& Litao W., 2007. Air pollution and its control in China. Frontiers of Environmental Science \& Engineering China, 1, 2, 129142.

Kan H., Chen R. \& Tong S., 2012. Ambient air pollution, climate change and population health in China. Environment International, 42, 10-19.

Kiebała A., Kozieł M. \& Zgłobicki W., 2015. Cr, Cu, Ni, Pb i $\mathrm{Zn} \mathrm{w}$ pyle drogowym na terenie Lublina. Inżynieria i Ochrona Środowiska, 18, 3, 299-310.

Krajewska E. \& Niesiobędzka K., 2009. Wpływ zasolenia spływów powierzchniowych na wymywanie metali z pyłów ulicznych do fazy wodnej. Ochrona Środowiska i Zasobów Naturalnych, 40, 137-143.

Kurt-Karakus P.B., 2012. Determination of heavy metals in indoor dust from Istanbul, Turkey: Estimation of the health risk. Environment International, 50, 47-55.

Lee H., Honda Y., Hashizume M., Guo Y.L., Wu C.F., Kan H., Jung K., Lim Y.H., Yi S. \& Kim H., 2015. Short-term exposure to fine and coarse particles and mortality: a multicity time-series study in East Asia. Environmental Pollution, 207, 43-51.

Lemly A.D., 1996. Evaluation of the hazard quotient method for risk assessment of selenium. Ecotoxicology and Environmental Safety, 35, 156-162.

Li P., Xin J., Wang Y., Li G., Pan X., Wang S., Cheng M., Wen T., Wang G. \& Liu Z., 2015. Association between particulate matter and its chemical constituents of urban air pollution and daily mortality or morbidity in Beijing City. Environmental Science \& Pollution Research, 22, 358-368.

Lippmann M., Chen L.C., Gordon T., Kazuhiko I., Thurston G.D., 2013. National Particle Component Toxicity (NPACT) initiative: integrated epidemiologic and toxicologic studies of the health effects of particulate matter components. Research Report Health Effects Institute, 177, 5-13.

Liu E., Yan T., Birch G. \& Zhu Y., 2014. Pollution and health risk of potentially toxic elements in urban road dust in Nanjing, a mega-city of China. Science of the Total Environment, 476-477, 522-531.

Megido L., Suárez-Peña B., Negral L., Castrillón L., Suárez S., Fernández-Nava Y. \& Marañón E., 2016. Relationship between physico-chemical characteristics and potential toxicity of PM10. Chemosphere, 162, 73-79.

Mohmand J., Eqani S.A.M.A.S., Fasola M., Alamdar A., Mustafa I., Ali N, Liu L., Peng S. \& Shen H., 2015. Human exposure to toxic metals via contaminated dust: Bio-accumulation trends and their potential risk estimation. Chemosphere, 132, 142-151.

Munir S., Gabr S., Habeebullah T.M. \& Janajrah M.A., 2016. Spatiotemporal analysis of fine particulate matter (PM2.5) in Saudi Arabia using remote sensing data. Egyptian Journal of Remote Sensing and Space Science.
Nag S., Gupta A.K. \& Mukhopadhyay U.K., 2005. Size distribution of atmospheric aerosols in Kolkata, India and the assessment of pulmonary deposition of particle mass. Indoor Built Environment, 14, 381-389.

Pant P., Guttikunda S.K. \& Peltier R.E., 2016. Exposure to particulate matter in India: A synthesis of findings and future directions. Environmental Research, 147, 480-496.

Patra A.K., Gautam S. \& Kumar P., 2016. Emissions and human health impact of particulate matter from surface mining operation - a review. Environmental Technology \& Innovation, 5, 233-249.

Peters A., Dockery D.W., Muller J.E. \& Mittleman M.A., 2001. Increased particulate air pollution and the triggering of myocardial infarction. Circulation, 103, 28102815.

Polichetti G., Cocco S., Spinali A. Trimarco V. \& Nunziata A., 2009. Effects of particulate matter (PM 10, PM2.5, PM1.0) on the cardiovascular system. Toxicology, 261, 1-8.

Pope III C.A., Burnett R.T., Thun M.J., Calle E.E., Krewski D., Ito K. \& Thurston G.D., 2002. Lung cancer, cardiopulmonary mortality and long-term exposure to fine particulate air pollution. Journal of American Medical Association, 287, 1132-1141.

Raes F., van Dingenen R., Vignati E., Wilson J. \& Putaud J.-P., 2000. Formation and cycling of aerosols in the global troposphere. Atmospheric Environment, 34, 4215-4240.

Rashki A., Eriksson P.G., de Rautenbach W.C.J., Kaskaoutis D.G., Grote W. \& Dykstra J., 2013. Assessment of chemical and mineralogical characteristics of airborne dust in the Sistan region, Iran. Chemosphere, 90, 227-236.

Rozporzadzenie Ministra Środowiska $z$ dnia 1 września 2016 r. w sprawie sposobu prowadzenia oceny zanieczyszczenia powierzchni ziemi. Dz.U. 2016, poz. 1395.

Samara C., Kantiranis N., Kollias P., Planou S., Kouras A., Besis A., Manoli E. \& Voutsa D., 2016. Spatial and seasonal variations of the chemical, mineralogical and morphological features of quasi-ultrafine particles (PM0.49) at urban sites. Science of the Total Environment, 553, 392-403.

Samet J.M., Dominici F., Curriero F.C., Coursac I. \& Zeger S.L., 2000. Fine particulate air pollution and mortality in 20 US cities 1987-1994. Journal of Medicine, 343, 1742-1749.

Sanderson P., Delgado-Saborit J.M. \& Harrison R.M., 2014. A review of chemical and physical characterisation of atmospheric metallic nanoparticles. Atmospheric Environment, 94, 353-365.

Sarigiannis D.A., Karakisios S.P., Zikopulos D., Nikolaki S. \& Kermenidou M., 2015. Lung cancer risk from PAHs emitted from biomass combustion. Environmental Research, 137, 147-156.

Shao M., Tang X., Zhang Y. \& Li W., 2006. City clusters in China: air and surface water pollution. Frontiers in Ecology and the Environment, 4, 7, 353-361.

Tomaszewska B. \& Olszowski T., 2012. Ilościowa i jakościowa ocena depozycji pyłu na obszarze wsi. Proceedings of ECOpole, 6, 2, 609-616.

Trojanowska M. \& Świetlik R., 2016. Ocena narażenia mieszkańców miast na metale ciężkie obecne w pyłach ulicznych. Bezpieczeństwo i Ekologia, Autobusy, 12/2016, 474-478. 
US EPA, 1989. Risk Assessment Guidance for Superfund. Vol. 1: Human Health Evaluation Manual. EPA/540/189/002. Office of Solid Waste and Emergency Response.

US EPA, 1996a. Method 3050B: Acid Digestion of Sediments, Sludges, and Soils. Revision 2.

US EPA, 1996b. Soil Screening Guidance: Technical Background Document. EPA/540/R-95/128. Office of Solid Waste and Emergency Response.

US EPA, 1998. Method 6020A (SW-846): Inductively Coupled Plasma-Mass Spectrometry. Revision 1.

US EPA, 2001. Supplemental Guidance for Developing Soil Screening Levels for Superfund Sites. OSWER 9355.4-24. Office of Solid Waste and Emergency Response.

US EPA, 2016. Regional Screening Level (RSL). Summary Table (TRD1E-6, HQD1). May 2016.

Wang M., Beelen R., Stafoggia M. et al., 2014. Long-term exposure to elemental constituents of particulate matter and cardiovascular mortality in 19 European cohorts: results from the ESCAPE and TRANSPHORM projects. Environment International, 66, 97-106.

WHO, 2013. Health Effects of Particulate Matter. Policy Implications for Countries in Eastern Europe. World Health Organization, Caucasus and Central Asia.
WHO, 2016. WHO ambient air pollution database. May 2016.

Wilczyńska-Michalik W. \& Michalik M., 2015. Skład i pochodzenie cząstek pyłów w powietrzu atmosferycznym w Krakowie. Aura, 3, 12-16.

Wilczyńska-Michalik W., Rzeźnikiewicz K., Pietras B. \& Michalik M., 2014. Fine and ultrafine $\mathrm{TiO}_{2}$ particles in aerosol in Kraków (Poland). Mineralogia, 45, 3-4, 65-77.

Wilczyńska-Michalik W., Pietras B., Samek L., Furman L., Łatkiewicz A., Rzeźnikiewicz K. \& Michalik M., 2015. Submikronowe pyły $\mathrm{w}$ powietrzu atmosferycznym w Krakowie. Aura, 8, 4-7.

Williams J., de Reus M., Krejci R., Fischer H. \& Ström J., 2002. Application of the variability-size relationship to atmospheric aerosol studies: estimating aerosol lifetimes and ages. Atmospheric Chemistry and Physics, 2, 133-145.

WIOŚ, 2017. Wojewódzki Inspektorat Ochrony Środowiska w Krakowie, http://monitoring.krakow.pios.gov.pl/.

Zhou M., He G., Liu Y., Fischer H. \& Ström J., 2015. The associations between ambient air pollution and adult respiratory mortality in 32 major Chinese cities, 20062010. Environmental Research, 137, 278-286. 